\title{
The Science Program of the TCV Tokamak: Exploring Fusion Reactor and Power Plant Concepts
}

\author{
S. Coda for the TCV team \\ Ecole Polytechnique Fédérale de Lausanne (EPFL), Centre de Recherches en Physique des \\ Plasmas, CH-1015 Lausanne, Switzerland
}

E-mail contact of main author: $\underline{\text { stefano.coda@epfl.ch }}$

\begin{abstract}
TCV is acquiring a new $1 \mathrm{MW}$ neutral beam and $2 \mathrm{MW}$ additional ECRH to expand its operational range. Its existing shaping and ECRH launching versatility was amply exploited in an eclectic 2013 campaign. A new sub-ms real-time equilibrium reconstruction code was used in ECRH control of NTMs and in a prototype shape controller. The detection of visible light from the plasma boundary was also successfully used in a position-control algorithm. A new bang-bang controller improved stability against vertical displacements. The RAPTOR real-time transport simulator was employed to control the current density profile using ECCD. Shot-byshot internal inductance optimization was demonstrated by iterative learning control of the current reference trace. Systematic studies of suprathermal electrons and ions in the presence of ECRH were performed. The L-H threshold power was measured to be $\sim 50 \%$ higher in both $\mathrm{H}$ and $\mathrm{He}$ than $\mathrm{D}$, to increase with the length of the outer separatrix, and to be independent of the current ramp rate. Core turbulence was found to decrease from positive to negative edge triangularity deep into the core. The geodesic-acoustic mode was studied with multiple diagnostics, and its axisymmetry was confirmed by a full toroidal mapping of its magnetic component. A new theory predicting a toroidal rotation component at the plasma edge, driven by inhomogeneous transport and geodesic curvature, was tested successfully. A new high-confinement mode (IN-mode) was found with an edge barrier in density but not in temperature. The edge gradients were found to govern the scaling of confinement with current, power, density, and triangularity. The dynamical interplay of confinement and MHD modes leading to the density limit in TCV was documented. The heat flux profile decay lengths and heat load profile on the wall were documented in limited plasmas. In the snowflake (SF) divertor configuration the heat flux profiles were documented on all four strike points. SF simulations with the EMC3-EIRENE code, including the physics of the secondary separatrix, underestimate the flux to the secondary strike points, possibly resulting from steadystate $\mathrm{E} \times \mathrm{B}$ drifts. With neon injection, radiation in a SF was $15 \%$ higher than in a conventional divertor. The novel triple-null and X-divertor configurations were achieved transiently in TCV.
\end{abstract}

\section{Introduction}

The Plasma Physics Research Center (CRPP) of the Federal Institute of Technology in Lausanne (EPFL) coordinates the entire Swiss contribution to experimental research in magnetic nuclear fusion. The Tokamak à Configuration Variable (TCV) [1] is its flagship facility. It has been in operation since 1992 and occupies a crucial position in the European fusion landscape as one of three national tokamaks supported by the newly established EUROfusion consortium for the Horizon 2020 research program (2014-2020). From 2015 TCV will be partly operated as a European facility under the auspices of the EUROfusion Medium-Size Tokamak (MST) Task Force. The flexibility and versatility of its magnetic-coil and auxiliary-heating systems, as well as the adaptability of its operational environment, underpin its role as a test bed of novel concepts, instruments, and techniques over a broad range of regimes and configurations. The experimental program of TCV is constructed primarily to address open issues related to the first envisioned incarnation of a fusion reactor, ITER, as well as more general scientific and technological questions for next step devices or a prototype fusion power plant such as DEMO. However, in the context of its internal program and in keeping with its academic mission, ample time is always allocated also to more fundamental plasma-physics problems.

The main operational parameters of TCV are the following: major radius $0.88 \mathrm{~m}$, minor radius $0.25 \mathrm{~m}$, vacuum toroidal field up to $1.5 \mathrm{~T}$, plasma current up to $1 \mathrm{MA}$, elongation up to 2.8 , triangularity from -0.7 to 1 . This uniquely expansive shaping capability extends to limited and diverted plasmas, the latter featuring an open divertor geometry and including 

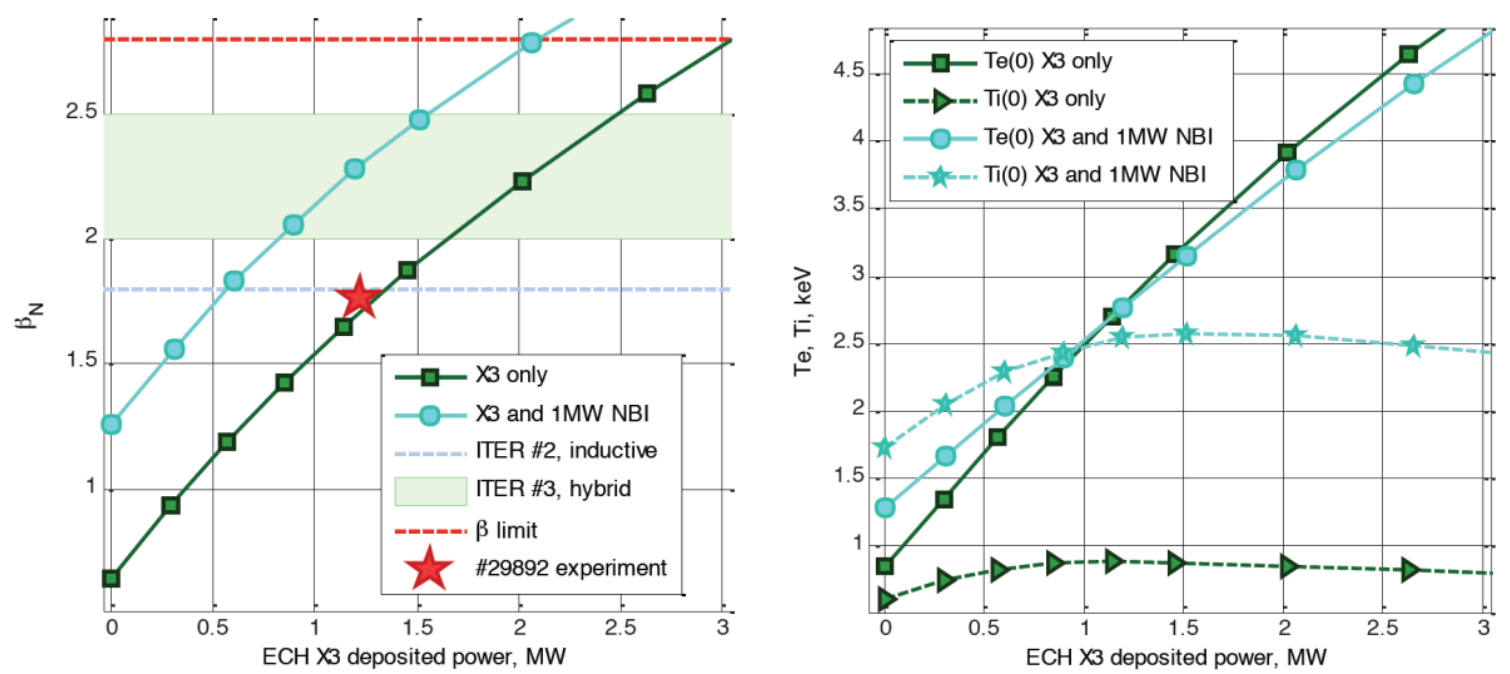

FIG. 1. ASTRA simulation of (left) normalized $\beta$ and (right) central electron and ion temperatures as functions of the X3 power, based on an H-mode shot with $I_{p}=410 \mathrm{kA}, B_{T}=1.44 \mathrm{~T}, q_{95}=2.5, Z_{\text {eff }}=2.6$, $n_{e}(0)=6.5 \times 10^{19} \mathrm{~m}^{-3}, T_{e}(0)=2.8 \mathrm{keV}, T_{i}(0)=0.9 \mathrm{keV}$, assuming a $30 \mathrm{keV} D$ beam and using the ITER H98 confinement scaling with $\chi_{i}=\chi_{e} / 2$.

single- and double-null as well as snowflake topologies. Auxiliary heating up to now was supplied exclusively in the form of Electron Cyclotron Resonance Heating (ECRH), at both the second (X2) and third (X3) harmonics, through seven independently steerable antennas, with a historical peak delivered power of $\sim 4 \mathrm{MW}$ (currently down to $2.5 \mathrm{MW}$ ) [2]. Standard operational scenarios include L-mode, H-mode, Internal Transport Barriers (ITBs), and steady-state non-inductive operation.

The TCV team has embarked upon an ambitious upgrade program, including $1 \mathrm{MW}$ neutral beam heating (NBH) by 2015, $1.5 \mathrm{MW}$ additional X2 power by 2016 (to replace end-of-life equipment loss), and $2 \mathrm{MW}$ additional switchable dual-frequency $\mathrm{X} 2 / \mathrm{X} 3$ power by 2018 [3]. The former in particular is designed to provide direct ion heating, to bridge the gap between the traditional $T_{e} \gg T_{i} T C V$ domain and the burning-plasma $\mathrm{T}_{\mathrm{e}} \sim \mathrm{T}_{\mathrm{i}}$ regime, with a sizeable fast-ion population. The additional $\mathrm{X} 2 / \mathrm{X} 3$ ECRH power is designed to attain an ITER-relevant regime of low collisionality and high $\beta$, as well as to reliably access advanced scenarios such as the quasi-stationary ELM-free H-mode [4]. The X3 waves can penetrate plasmas at densities up to $1.2 \times 10^{20} \mathrm{~m}^{-3}$, compatible with H-mode, and as shown in Fig. 1 the combination of $\mathrm{NBH}$ and $\mathrm{X} 3 \mathrm{ECRH}$ is expected to lift the plasma $\beta_{\mathrm{N}}$ to 2.8 , well into the ITER range, spanning the range $\mathrm{T}_{\mathrm{e}} / \mathrm{T}_{\mathrm{i}}=0.5-3[5]$.

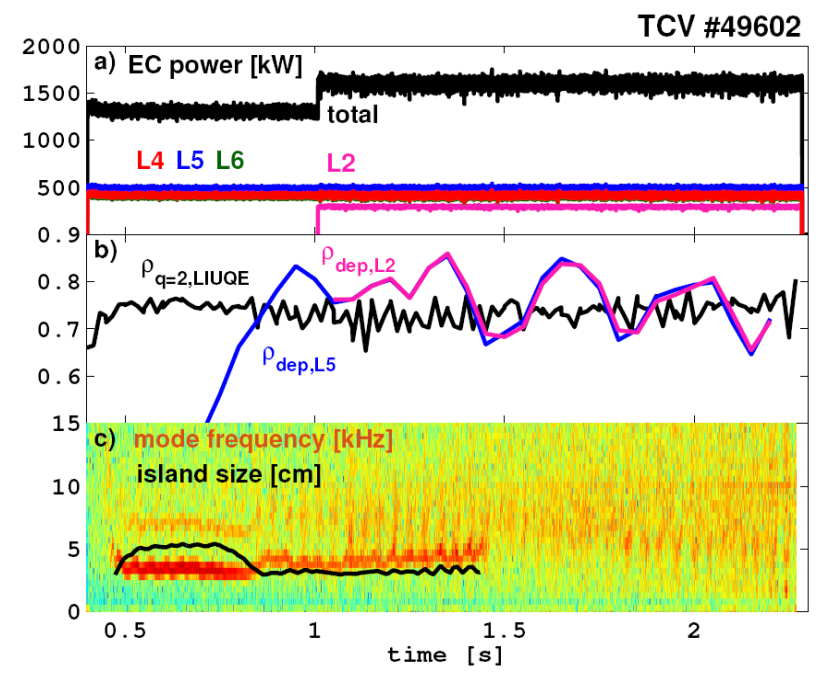

FIG. 2. Demonstration of robust NTM stabilization. Top: ECRH power, including central power to destabilize the NTM $(L 4+L 6)$ and power applied near the $q=2$ surface $(L 2+L 5)$; center: radial locations of $q=2$ surface and off-axis power deposition; bottom: MHD spectrogram and mode amplitude. With sufficient power, the mode is stabilized on the first oscillation cycle as the power hits the $q=2$ surface. (The oscillation here is centered around the $q=2.5$ surface to test robustness against errors.) 
The neutral-beam injector and geometry, which features tangential injection with two passes through the plasma column, were optimized for minimum shine-through and orbit losses. The main parameters are a tunable energy from 18 to $30 \mathrm{keV}$, tunable power from 0.3 to $1 \mathrm{MW}$ for up to $2 \mathrm{~s}$, and operability in deuterium or hydrogen [6]. The new X2/X3 gyrotrons are based on the European development of 1-MW, 170-GHz triode sources for ITER but will be designed to operate at 84 and $126 \mathrm{GHz}$ [7].

This paper reports on the scientific work performed on TCV in an eclectic and intensive 2013 campaign, before the 2014 shutdown for NBH preparation and installation. The remainder of the paper is organized by subject, as follows: section 2, real-time control; 3, ECRH physics; 4, H-mode and ELMs; 5, core MHD; 6, microturbulence; 7, spontaneous rotation and momentum transport; 8, energy and particle confinement; 9, edge physics and advanced exhaust concepts. Concluding remarks are offered in section 10.

\section{Real-time plasma control}

The legacy analogue plasma control system (PCS) of TCV is progressively being replaced by a recently commissioned, distributed digital system [8,9]. The latest campaign has especially benefited from the development of a real-time version of our in-house Grad-Shafranov equilibrium reconstruction code, LIUQE. The complete real-time cycle (RTLIUQE), including flux contour identification and a calculation of flux-surface-averaged quantities, requires 200 $\mu$ s [10].

A natural application of RTLIUQE was the extension and refinement of previous work on the stabilization of neoclassical tearing modes (NTMs) by ECRH applied to the relevant rational safety-factor $(q)$ surface [11,12]. Using a pre-calculated lookup table linking the minor radius $\rho$ with the launcher angle, the latter is actuated based on the real-time determination of the rational surface's location [13]. To obviate uncertainties in the reconstruction, an additional sinusoidal oscillation is superimposed on the launcher movement, increasing the probability that the appropriate target is hit at some point during the cycle and enhancing the robustness of the control strategy [14]. This applies to both pre-emption, which requires less power, and stabilization of the mode - a backup solution should it nevertheless be triggered (Fig. 2). The efficacy of this novel control strategy has recently been confirmed on ASDEX Upgrade [15].

The NTM pre-emption and stabilization strategies demonstrated on TCV, the former extending also to the pacing of the sawtooth cycle that is the most likely NTM trigger, have been recently examined also for their applicability to ITER, with encouraging conclusions [16].

RTLIUQE has also been deployed as an input to a prototype, generalized shape and position controller, using an isoflux algorithm applied to a discrete set of points on the plasma boundary. An initial demonstration succeeded in reducing the flux errors beyond those achieved by the standard feedforward technique [17].

We have pursued in parallel a demonstration of position control relying on visible-light images of the plasma edge. The OFIT algorithm was developed to reconstruct the 2D boundary contour from images obtained through a wide-angle, tangential view of the plasma column, based on predetermined regions of interest. The vertical position estimation by this method was shown to agree to within $1 \mathrm{~cm}(\sim 3 \%$ of the minor radius) with the offline equilibrium reconstruction. Real-time vertical position control was also successfully demonstrated [18]. The stabilization of the axisymmetric MHD instability of highly elongated plasmas requires faster response than can be provided by reconstruction algorithms. The internal stabilization coils of TCV are designed to counter growth rates of up to $3000 \mathrm{~s}^{-1}$. A bang-bang controller was developed in an effort to improve even further over the performance of the analogue PCS. This was implemented in a set of digital signal processors and tested successfully on a 
discharge featuring an elongation ramp: higher elongation was achieved by the new controller than by the standard one [19].

Feedback control of the plasma breakdown is a future aim of the TCV program to increase the success rate over the current empirical strategy. To this end, parameter scans were performed and analyzed with a model employing the full complement of magnetic measurements and including the time derivatives of all poloidal-field coil currents and a calculation of the conducting vessel's eddy currents, which can reach up to $200 \mathrm{kA}$. The model identifies breakdown locations that are in good agreement with inverted visible camera images, providing a sound basis for controller design [20].

Significant resources are being invested worldwide in the development of tokamak current density profile controllers. Several such controllers have been linked with the RApid Plasma Transport simulatOR (RAPTOR) [21,22], a modular real-time $(<5 \mathrm{~ms})$ code for the computation of 1D $q$ and $T_{e}$ plasma profiles, for offline testing [23] as well as for eventual experimental verification on TCV. Initial closed-loop tests occurred during the last campaign. An alternative strategy relying on Iterative Learning Control, an offline method especially suited for repetitive tasks, was tested on TCV to optimize the internal inductance of the plasma (a measure of current-profile width) by tailoring the time trace of the plasma current. The proofof-principle test was successful in that satisfactory convergence was achieved over several discharges.

\section{ECRH physics}

The physics of ECRH and particularly ECCD is mediated by the acceleration of electrons to potentially suprathermal energies, especially with the high power densities present in TCV. A tomographic hard X-ray spectrometer (HXRS) has been developed [24] to study the dynamics of suprathermal electrons through their bremsstrahlung emission and has recently generated first results from three of the eventual four cameras. Using advanced digital pulse recognition techniques [25], the first tomographic inversions of HXR data from a shaped tokamak plasma have now been produced. Initial analysis indicates that the poloidal asymmetry of HXR emission from EC-heated plasmas is in only partial agreement with quasilinear predictions processed by a synthetic diagnostic.

Earlier tests of the feasibility of the detection of escaped microwave radiation as a measure of the correctness of the wave polarization have undergone further analysis, revealing discrepancies with ray-tracing predictions, which however do not appear to preclude the use of this technique for real-time polarization control [14], which is a goal for ITER.

The long-standing question of the origin of a suprathermal ion tail observed with ECRH was brought closer to an answer in this campaign, thanks to new measurements of the ion distribution function with a tangential neutral particle analyzer at high EC power. The generation mechanism put forward for this phenomenon is a transfer of energy from weakly relativistic electrons to the ion population, mediated by ion acoustic turbulence [26]. This effect would be asymmetric in the direction parallel to the magnetic field, and this asymmetry was verified through reversal of the waves' toroidal wave number as well as of the plasma current.

\section{H-mode and ELMs}

The power threshold for access to H-mode has been studied amply in deuterium plasmas, but much less with alternative species, such as hydrogen or helium. The issue has garnered heightened attention lately as operation in $\mathrm{H}$ or $\mathrm{He}$ is envisioned for the initial, non-nuclear operation phase of ITER, and existing data are not sufficient to ascertain with confidence whether the available power will be sufficient to attain H-mode [27]. An investigation of the threshold dependence on power was therefore undertaken in TCV in Ohmic D, H, and $\mathrm{He}$ 


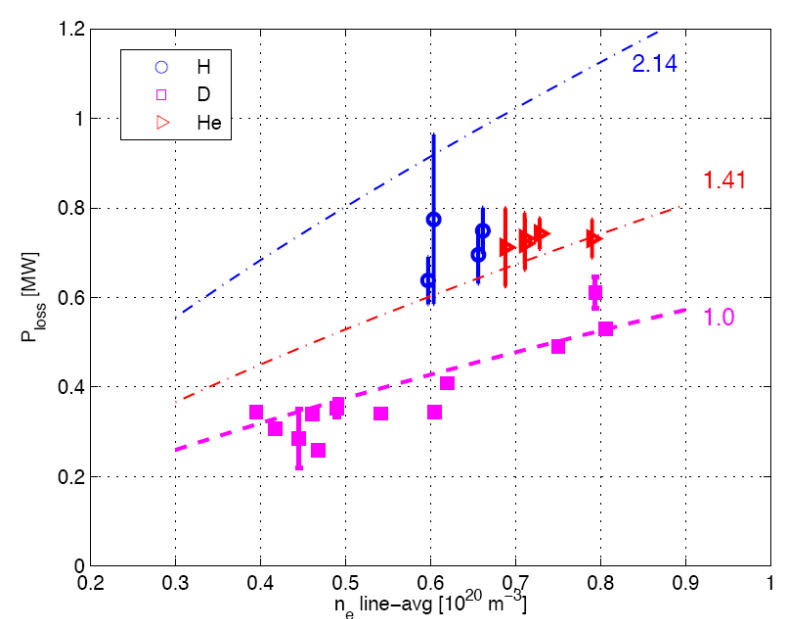

FIG. 3. Experimental Ohmic H-mode threshold power vs line-averaged density, compared with empirical scaling. (Reproduced from [28]). plasmas. The threshold power for $\mathrm{H}$ and $\mathrm{He}$ is found to exceed that for $\mathrm{D}$ by approximately $50 \%$ and $75 \%$, respectively [28]. This is broadly consistent with former results in the case of $\mathrm{He}$ but not in the case of $\mathrm{H}$, for which the threshold quoted in the literature is typically a factor of 2 higher than for D [29]. The leading international empirical scaling law [30], corrected for species dependence according to [31], also fits the $\mathrm{D}$ and $\mathrm{He}$ data but fails for $\mathrm{H}$, as shown by Fig. 3. The same holds for the physics-based scaling proposed in [32].

Other parametric dependences of the threshold were explored recently, again with an eye to ITER needs. In view of the possibility of entering $\mathrm{H}$-mode during the current ramp, the dependence on the ramp rate was examined, but none was found [33]. A strong increasing dependence on the other hand was evinced for the threshold as a function of the length of the poloidal projection of the lowfield-side divertor leg, by a factor of 2 when going from 10 to $35 \mathrm{~cm}$ [33].

In the general quest to discover effective means of ELM mitigation, advancing our relatively primitive physics understanding of ELMs plays an important and often underrated role. A recent study on TCV focused on the nonlinear phase of type-I ELMs, using a complete set of magnetic measurements [34]. The rigidity of the observed toroidal mode structure leads to the conclusion that the collected data pertained exclusively to the nonlinear phase, implying that the linear phase was too rapid to be accessible to measurements. Furthermore, a dominant $n=1$ toroidal mode number is found, while the peeling-ballooning modes believed to be responsible for ELMs are predicted to possess higher periodicity. This suggests a spatial cascade mechanism in the nonlinear evolution. The more global nature of the nonlinearlygenerated low- $n$ modes may help explain the large energy losses of type-I ELMs.

\section{Core MHD modes}

The deleterious effect of Neoclassical Tearing Modes (NTMs) on confinement has spurred extensive research on these instabilities over the past two decades. The most recent TCV campaign has contributed to this body of research in a study of the complex interplay of NTMs, plasma rotation, and ECRH. The focus was on NTMs excited by mechanisms other than sawtooth crashes, ELMs, or error fields, and particularly on the question of their possible destabilization by the ion polarization current alone without the appearance of seed islands. In the collisionless regime and in the absence of sizable sawteeth, power ramps of core coECCD drive an $(m, n)=(3,2)$ tearing mode unstable [35]. During the ramp, an increase towards the co-current direction of the spontaneous toroidal velocity of the plasma is also observed. The $(3,2)$ mode is followed by a further modification of the rotation profile, which flattens, and then by the appearance of a $(2,1)$ mode and the concomitant disappearance of the $(3,2)$ mode; further rotation flattening ensues. The direct effect of the EC waves on rotation is hypothesized to be due to a radial current generated by slight non-uniformities in the EC beam [36]. The change in the difference between the rotation frequency and the mode frequency can enhance the destabilizing effect of the ion polarization current and trigger the $(3,2)$ mode. However, the magnetic shear modification due to ECCD can also increase $\Delta^{\prime}$ and destabilize a conventional tearing mode, which then acts as the seed for the NTM; this is 
supported by the dependence of the timing of the mode appearance on the net ECCD component [35]. The reactive effect of the tearing mode on the rotation is believed to be mediated by neoclassical toroidal viscosity generated by the axisymmetry breaking effect of the mode [37].

The interplay between MHD instabilities and suprathermal electrons bears on fundamental properties of magnetic reconnection as well as on highly practical concerns related to runaway electrons and disruptions in a reactor. Studies have commenced using the new HXRS diagnostic, focusing thus far on long-lived - though bursty - core modes. Statistical analysis has shown that the ECCD-generated suprathermal population is sloshed radially by a $(1,1)$ mode but is essentially unaffected by a concomitant $(2,1)$ mode [38].

Recent theoretical work has predicted the possible existence of bifurcated MHD equilibrium states, one of which in particular possesses a helical structure resembling a saturated internal kink and is speculated to be an explanation for the "snake" structures reported by several devices [39]. The bifurcation requires weak negative core magnetic shear and a far off-axis safety-factor minimum near unity, as well as a peaked pressure profile [40]. A search for this equilibrium state was undertaken in TCV based on device-specific simulations. The strategy entailed current ramps with strong off-axis heating to delay the current penetration. A mode with the predicted characteristics was indeed triggered some time before the sawtooth cycles began, signaling the appearance of a $q=1$ surface. Detailed analysis is underway.

\section{Plasma microturbulence}

Studies of plasma fluctuations have gained momentum recently in TCV following the development or acquisition of several diagnostics [41]. These include a tangential phasecontrast imaging (TPCI) system for density fluctuations [42], a correlation ECE (CECE) system for temperature fluctuations [43], and a multi-frequency Doppler backscattering (DBS) system for flow measurements [44]. A particular focus that is natural to TCV has been the dependence of microturbulence, measured in the ITG-TEM spectral region, on edge triangularity $\delta$ and effective collisionality $v_{\text {eff }}$, following work that showed a strong dependence of transport on these quantities: the electron energy confinement increases from positive to negative $\delta$ and increases with $v_{\text {eff }}$ [45]. Initial results from a prototype 2-channel CECE, reported earlier, have now been extended with an upgraded, tunable 6-channel system. The main finding is that the relative temperature fluctuation level, concentrated spectrally in the $20-150 \mathrm{kHz}$ range, increases when going from negative to positive $\delta$ at constant input power in the entire radial range covered $(0.3<\rho<0.8)$, the difference being more pronounced near the edge; however, the absolute level, as well as the radial correlation length, is essentially unchanged [41,46]. The 9-channel TPCI apparatus also observes the relative fluctuation level to increase with $\delta$ and minor radius and to decrease with collisionality [47]. The triangularity effect, again, is seen deep into the core (to $\rho=0.5$ ). This may clash with the observation that the local triangularity is nearly vanishing there, that flux-tube gyrokinetic simulations accordingly fail to reproduce the dependence, and that the local gradients are also essentially independent of $\delta$ [48]. Further studies, as well as global gyrokinetic modeling and appropriate synthetic diagnostics, are required to gain further insight into these results.

A unique multi-diagnostic study of the Geodesic Acoustic Mode (GAM) was performed on TCV [49]. The GAM is detected as a coherent mode simultaneously by TPCI, CECE, DBS, and magnetic diagnostics, and is identified by its frequency scaling with the ion sound speed and by its axisymmetry. The latter was confirmed for the first time by a complete (16-point) toroidal mapping of its magnetic component. The simultaneous multi-system detection has allowed a determination of most relevant properties of the GAM, including its frequency, all components of its wave number, its radial distribution, and its associated flow velocity [49]. 


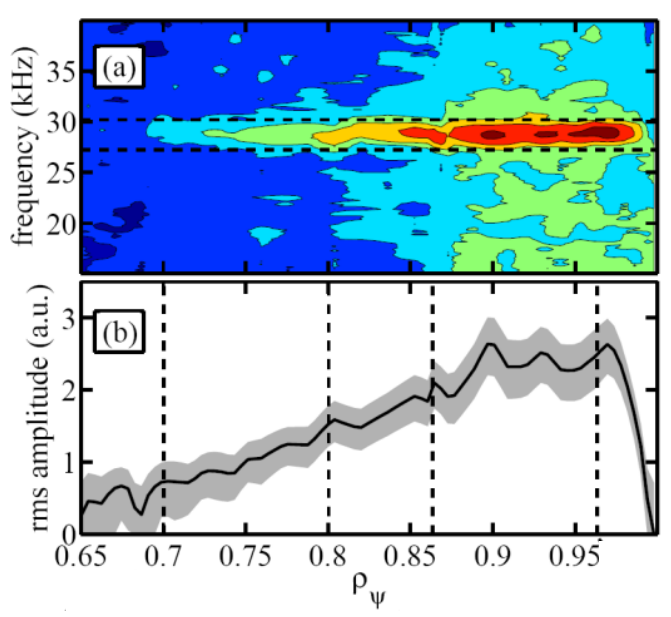

FIG. 4. TPCI signal: (a) autopower spectrum, (b) radial rms amplitude profile. (Adapted from [49]).

The magnetic component is found to have the dominant $m=2$, standing-wave poloidal structure predicted by theory [50]. The radial distribution of the GAM is broad (Fig. 4) and the frequency is spatially uniform in cases in which the magnetic signal is strong, indicating that the GAM is a global eigenmode. A simulation with the PIC gyrokinetic code ORB5 successfully reproduced all the main features of this mode [49]. In other cases, however, a dispersive continuum is seen as a function of minor radius, with the frequency scaling with the local sound speed. A transition between the two regimes has been observed directly in a TCV shot by means of a plasma current ramp: the eigenmode started fragmenting once $q_{95}$ climbed above $~ 6$ [47]. The drive and damping mechanisms of the GAM were tentatively probed with the aid of a density ramp. The GAM amplitude is found to be roughly independent of density whereas the broadband turbulence increases with it [47]. As the latter is believed to drive the GAM - as corroborated by a strong bicoherence level - it may be speculated that the damping increases with density as well, compensating the drive enhancement. This would support the hypothesis that the GAM is primarily damped by collisions, although Landau damping may also play a role.

\section{Plasma rotation and momentum transport}

Continuous improvements in the charge exchange recombination spectroscopy (CXRS) diagnostic [51] have kept pace with our ambition to obtain a full characterization of spontaneously generated (torqueless) toroidal and poloidal flows and of a closely related quantity, the radial electric field. A recent study collected a full $2 \mathrm{D}$ poloidal map of the temperature, density, and flow velocity of the main impurity, carbon. Good spatial resolution was achieved by slight vertical sweeps of the plasma past the fixed CXRS emission volumes. The impurity temperature is found to be poloidally symmetric, whereas the density is higher on the low-field side (LFS), and the toroidal flow velocity - which is indeed not expected to be a flux function - is also higher in absolute value on the LFS.

Continuing in a tradition of mobilizing resources to rapidly test new ideas, TCV was employed to test a new theory of edge toroidal rotation generation [52]. The putative source is the orbit asymmetry of co- and counter-propagating ions in the presence of turbulence, effectively resulting in inhomogeneous momentum transport. A key prediction is that the induced velocity would be linearly dependent on the major radius of the $\mathrm{X}$-point and change sign (to counter-current) for a sufficiently outward location [52]. This test was promptly carried out, and good agreement was found (Fig. 5).

In an application of performance-driven control techniques to diagnostic goals, the effect of sawtooth oscillations on momentum transport was studied with

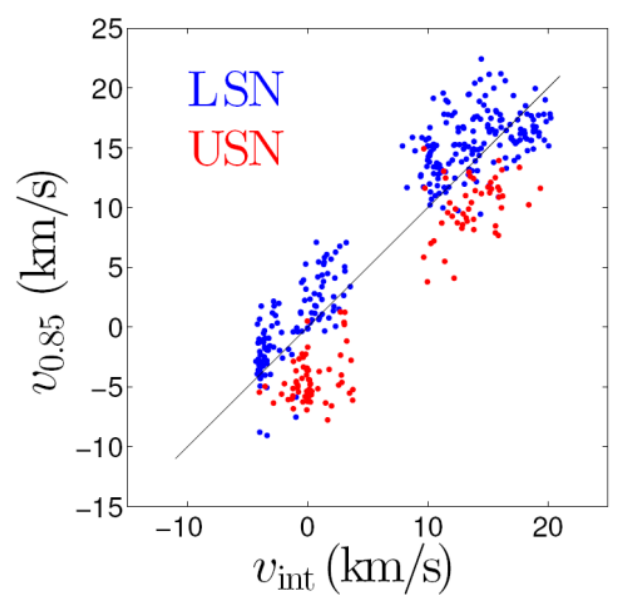

FIG. 5. Toroidal velocity at $\rho=0.85 \mathrm{vs}$ velocity predicted by model from [52] for lower- and upper-single-null plasmas with varying $X$-point major radius. 
greater effective time resolution than ever before, using coherent averaging over a large number of sawteeth [51], whose period was locked to modulated ECRH deposited near the $q=1$ surface [53]. The very rapid central spin-up at the sawtooth crash, hinted at by past measurements, is now clearly confirmed, as is the slower relaxation - nonetheless faster than the sawtooth period - to the pre-crash profile [51].

\section{Energy and particle confinement}

A new high-confinement mode was discovered recently in TCV, featuring L-mode-like electron temperature profiles but high edge density gradients and H-mode-like confinement, with H98Y2 0.8-1 (see Fig. 6). Dubbed IN-mode, this regime has been obtained thus far in Ohmic conditions through two alternative empirical paths: by starting the initial current rampup at high density and low internal inductance; or, at higher current with Ohmic power marginally above the H-mode threshold, by the plasma transiting to $\mathrm{H}$-mode and then reverting spontaneously to an enhanced L-mode. The ultimate steady-state profiles are similar in the two cases and the internal inductance is lower than in standard L-mode [54].

A dedicated campaign was devoted to a detailed characterization of the edge kinetic electron profiles (in the outermost $20 \%$ of the minor radius) in L-mode [48]. The resolution of the Thomson scattering system was effectively enhanced, again, by performing slow vertical sweeps of the plasma position. The pivotal result is that profiles are not "stiff" in this region, in the sense that the gradient scale lengths are neither spatially uniform nor parameterindependent; rather, the gradients themselves are spatially uniform and are approximately proportional to the plasma current as well as off-linearly dependent on the input power, whereas the value (of density, temperature, or pressure) at the plasma boundary changes little. By contrast, the confinement region - extending from the sawtooth inversion radius to $\rho \sim 0.8$ - features stiff profiles with uniform gradient lengths independent of current (see Fig. 7) or power. The stiffness of the core profiles is a well-documented property that was seen to imply that the global confinement is determined primarily by the value at the boundary. This new set of measurements effectively shifts this burden from the boundary to the entire $\rho \sim 0.8-1.0$ edge region, providing renewed motivation for understanding the physics underlying anomalous transport preferentially in this region. The same conclusion is found to apply to the confinement enhancement at negative triangularity $[48,54]$.

The dependence of confinement on density in the Linear Ohmic Confinement (LOC) regime also exhibits a similar behavior (as summarized by Fig. 8), with the edge gradients increasing

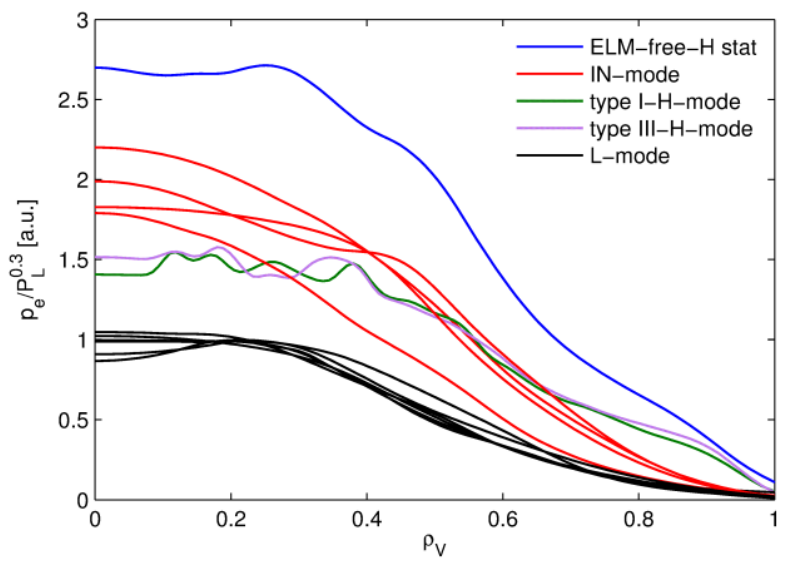

FIG. 6. H-factor profiles, i.e., electron pressure profiles divided by loss power and by the power scaling factor appearing in the L-mode confinement scaling, for several TCV regimes. linearly with density; however, the boundary density also increases [48]. The transition to the Saturated Ohmic Confinement (SOC) regime occurs typically when the lineaveraged density reaches $\sim 40 \%$ of the Greenwald limit $\left(\mathrm{n}_{\mathrm{GW}}\right)$. This is accompanied by a self-reorganization that leaves the electron pressure profile unchanged, while the central density increases and the central temperature decreases, lengthening the sawtooth period in the process. The approach to the density disruption limit generally sees a collapse of the edge temperature, an increase in core radiation, and the stabilization of sawteeth (at $0.6 \times \mathrm{n}_{\mathrm{GW}}$ ); this in turn leads to further density peaking and core 


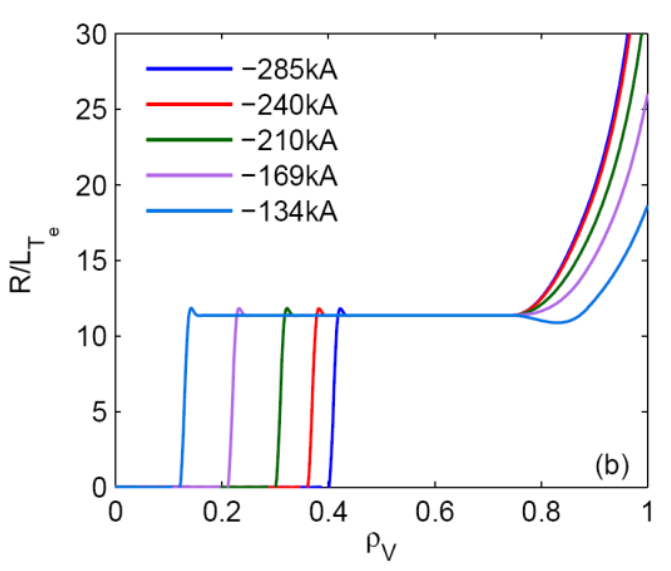

FIG. 7. Profiles of normalized electron temperature gradient length at different plasma currents, based on fits to a constant temperature inside the inversion radius, to an exponential outside it and up to $\rho=0.8$, and to a linear function for $\rho>0.8$. (Reproduced from [48]). radiation in a positive feedback cycle $[48,55,56]$. The $q$ profile then becomes unstable for the $(2,1)$ mode - generally transiting through the excitation and crash of a $(1,1)$ mode - which leads to further cooling and discharge termination. The relative limit density $\left(\left\langle\mathrm{n}_{\mathrm{e}}\right\rangle / \mathrm{n}_{\mathrm{GW}}\right)$ decreases with current and increases with triangularity (typically $\sim 0.65$ at $200 \mathrm{kA}$ and $\sim 1$ at $110 \mathrm{kA}$ at $\delta=+0.3,25 \%$ lower at $\delta=-0.3)[56]$.

The role of sawtooth stabilization in the attainment of the density limit was further highlighted by the application of core ECRH, which delays density peaking through a well-known pumpout effect and consequently delays the sawtooth stabilization, ultimately increasing the effective density limit for disruption [55].

Discharges with electron internal transport barriers sustained by off-axis ECCD were revisited in a quest to study ion confinement as well as the

behavior of the radial electric field. In these conditions with $T_{e} \gg T_{i}$ and poor energy transfer from electrons to ions, no barrier arises in the ion channel [57]. The measured $\mathrm{E} \times \mathrm{B}$ shearing rate is uncorrelated to the electron barrier strength, ruling it out as a significant factor in barrier formation. This is consistent with the shearing rate being approximately one order of magnitude smaller than the calculated growth rate of the dominant TEM instability [57]. We have also revisited our fully bootstrap-driven steady-state scenarios, confirming the robustness of the effect by steadily recharging the Ohmic transformer at constant plasma current.

\section{Edge physics and advanced exhaust concepts}

Edge physics in both conventional and advanced-shape configurations is an expanding chapter of our program. Data from JET have elicited concern that in the initial limited phase of ITER discharges the heat flux to the limiter may be higher than expected [58]. This concern was addressed in TCV Ohmic plasmas using infrared imaging in conjunction with the THEODOR code to derive the heat flux. The perpendicular heat flux, which increases with plasma current, accounts for $20 \%$ of the total, and the parallel flux is also enhanced near the contact point. While the perpendicular flux is well described by a single decay length $(2-8 \mathrm{~mm})$, the parallel flux features two decay lengths $(2-6 \mathrm{~mm}$ and $12-32 \mathrm{~mm})$. The calculated excess power entering the SOL from the narrower feature increases with temperature and decreases with density, current, and elongation [59].

In the snowflake (SF) divertor configuration, and in both the SF+ and SF- topological variants, we have now documented the heat flux on all four

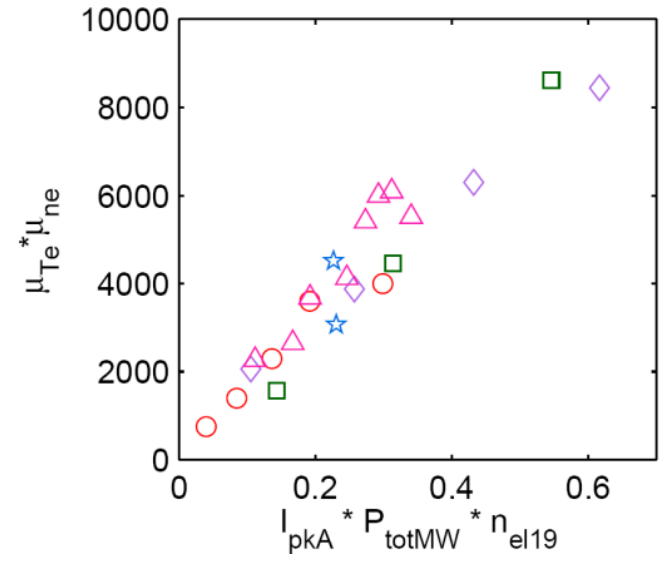

FIG. 8. Product of edge temperature and density gradients (in a.u.) as a function of the product of plasma current, input power, and line-averaged density for a variety of conditions (see [48] for details). (Reproduced from [48]). 
strike points with Langmuir probes (LPs) [60]. Infrared thermography is available for one primary and one secondary strike point [61], and tomographic foil bolometers as well as AXUV pseudo-bolometers [62] are used for radiation measurements. The peak heat fluxes are reduced with respect to a conventional single-null $(\mathrm{SN})$ divertor, and enhanced transport into the private flux region is inferred (Fig. 9). Significantly, the scrape-off layer (SOL) is not broader in the SF than in the SN regime, suggesting no increase in transport in the common flux region $[60,63]$. This is to be expected in contemporary tokamaks, whose size is inadequate to reap the full potential advantages of the SF topology: the poloidal field is lower and the connection length is longer than in the SN only very close to the X-point. However, projections to a DEMO-sized device indicate that the properties of a SF would affect it far more favorably: an increased divertor volume radiating more power, and a longer connection length helping detachment and possibly widening the SOL, whose intrinsic width scales less than linearly with device size [60].

The potential radiating properties of $\mathrm{SF}$ vs $\mathrm{SN}$ topologies were specifically investigated through density ramps. Consistent with the volume deficit, the SF radiates typically $10 \%$ less than the SN divertor at equal density in TCV. However, with Ne puffing the opposite occurs, with $\sim 15 \%$ more radiation from the SF. The difference is attributed to the different radiation loss parameter of $\mathrm{C}$ (the main intrinsic TCV impurity) and $\mathrm{Ne}$, which radiates at higher temperature, favoring the hotter null region over the outer SOL and thus benefiting from the local flux expansion there $[63,64]$.

Modeling of SF scenarios has now begun in earnest with the first SF+ simulations with the 3D edge Monte Carlo transport code EMC3-EIRENE, constraining the transport coefficients by imposing a match to the LP profiles. The simulation is unable to reproduce the measured degree of transport enhancement into the private flux region, underestimating the heat flux to the secondary strike points by one order of magnitude [65]. This could be related to the absence of particle drifts in the EMC3-EIRENE suite. Convection driven by toroidal curvature in the $\beta_{\mathrm{p}}>1$ region near the null point has been invoked to explain the excess transport [66].

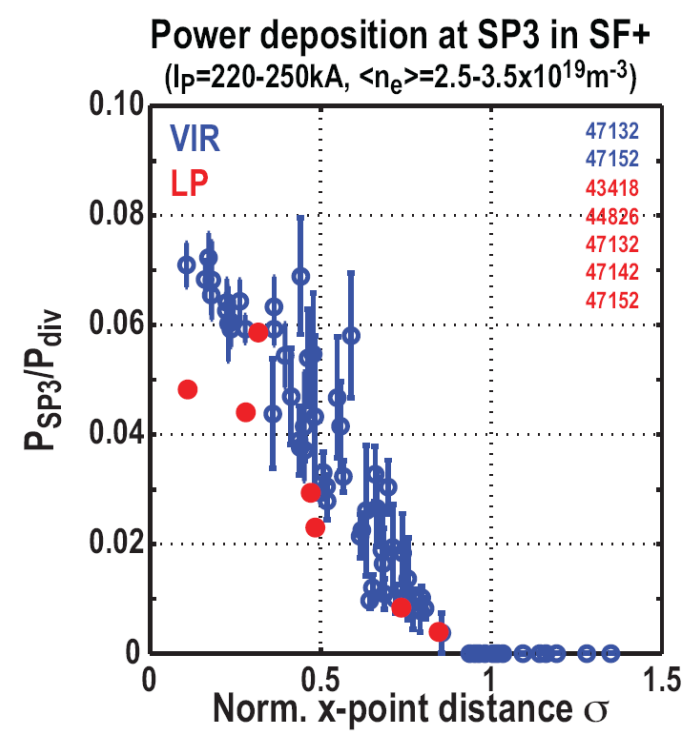

FIG. 9. Power deposition to the outer secondary strike point in a SF+ plasma, normalized to the total exhausted power, vs $X$-point separation: data from Langmuir probes (LP) and infrared thermography (VIR). (Reproduced from [60]).
More recently, estimates of the $\mathrm{E} \times \mathrm{B}$ drift in the null region have yielded fluxes well in excess of those calculated by EMC3-EIRENE, especially in the poloidal direction [67]. These drifts may also explain the second heat flux peak measured at high density on the main high-field side target, also visible in the SN but more pronounced in the SF case $[63,67]$.

Fluctuation measurements by LPs have revealed signs of intermittency or bursty behavior in both the SN and SF configurations, in the SOL as well as in the private flux region near the high-field-side primary strike point (and also on the LFS strike point in the case of the SF-) [63].

While a variety of SF configurations can comfortably be created in TCV, their parameter range remains limited by coil current limitations, either power-supply-related or safety-related in terms of coil stresses. Using the linear CREATE-L and nonlinear CREATE-NL equilibrium simulation codes to optimize the discharge design, a $20 \%$ increase in achievable SF plasma current was 
demonstrated [68].

Even more exotic configurations were recently attempted and achieved, at least transiently: a triple-null (3 X-points around the plasma boundary) and the so-called X-divertor, a conventional single-null shape with an exceptionally high flux expansion [69]. These initial studies open the way to further research avenues into future innovative exhaust solutions.

\section{Conclusions and outlook}

A highly diversified scientific campaign has taken place in TCV in 2013, aligned loosely along the three interconnected paths of reactor science, advanced concepts for power plants, and fundamental plasma physics. Highlights include robust NTM control using real-time equilibrium reconstruction; the determination of the H-mode threshold power for multiple species; a full multi-field characterization of the GAM; the experimental verification of a turbulence-driven mechanism for edge toroidal flow generation; the discovery of a new enhanced particle-confinement mode; the establishment of the role of the outermost $20 \%$ of the plasma column in governing global confinement; and a thorough study of heat fluxes to divertor targets in the snowflake topology, accompanied by first numerical simulations.

TCV is currently being fitted with a 1-MW neutral beam injector which signals the beginning of a new phase in its history, in which existing capabilities will be augmented by an ability to study the hot-ion, high- $\beta$ reactor realm in a timely fashion as the ITER horizon looms ever closer.

\section{Acknowledgments}

This work was supported in part by the Swiss National Science Foundation. This work has been carried out within the framework of the EUROfusion Consortium and has received funding from the European Union's Horizon 2020 research and innovation programme under grant agreement number 633053. The views and opinions expressed herein do not necessarily reflect those of the European Commission.

\section{References}

[1] HOFMANN, F., et al., Plasma Phys. Control. Fusion 36 (1994) B277.

[2] GOODMAN, T.P., and the TCV team, Nucl. Fusion 48 (2008) 054011.

[3] FASOLI, A., et al., this conference, FIP/P7-7.

[4] PORTE, L., et al., Nucl. Fusion 47 (2007) 952.

[5] KARPUSHOV, A.N., et al., Fusion Eng. and Design 86 (2011) 868.

[6] SOROKIN, A., et al., Rev. Sci. Instrum. 81 (2010) 02B108.

[7] ALBERTI, S., et al., Proc. $35^{\text {th }}$ Int. Conf. Infrared Millimeter and Terahertz Waves, Rome, 2010, IEEE.

[8] LE, H.B., et al., Fusion Eng. and Design 89 (2014) 155.

[9] FELICI, F., et al., Fusion Eng. and Design 89 (2014) 165.

[10] MORET, J.-M., et al., to be published in Fusion Eng. and Design (2014).

[11] FELICI, F., et al., Nucl. Fusion 52 (2012) 074001.

[12] GOODMAN, T.P., et al., AIP Conference Proceedings 1580 (2014) 161.

[13] KIM, D., et al., Proc. 55 ${ }^{\text {th }}$ DPP-APS Meeting, Denver, USA, Bull. Am. Phys. Soc. 58 (2013) PP8.00073.

[14] GOODMAN, T.P., et al., Proc. $18^{\text {th }}$ Joint ECE-ECRH Workshop (EC-18), Nara, Japan, 2014.

[15] REICH, M., et al., this conference, PPC/P1-26.

[16] KIM, D., et al., Phys. Plasmas 21 (2014) 061503.

[17] ANAND, H., et al., Proc. $41^{\text {st }}$ Conf. on Control. Fusion and Plasma Phys., Berlin, 2014, Europhys. Conf. Abstr. 38F (2014) (P5.069).

[18] HOMMEN, G., et al., Nucl. Fusion 54 (2014) 073018.

[19] CRUZ, N., et al., submitted to IEEE Trans. Nucl. Sci. (2014).

[20] SINHA, J., et al., Proc. $41^{\text {st }}$ Conf. on Control. Fusion and Plasma Phys., Berlin, 2014, Europhys. Conf. Abstr. 38F (2014) (P5.070).

[21] FELICI, F., et al., Nucl. Fusion 51 (2011) 083052.

[22] FELICI, F., et al., Proc. American Control Conf., Portland, OR, USA, 2014.

[23] BARTON, J., et al., submitted to Proc. 53 ${ }^{\text {rd }}$ IEEE Conf. on Decision and Control, Los Angeles, USA, 
2014.

[24] GNESIN, S., et al., Rev. Sci. Instrum. 79 (2008) 10 F504.

[25] KAMLEITNER, J., et al., Nucl. Instrum. and Methods in Phys. Res. A 736 (2014) 88.

[26] SCHLATTER, Ch., et al., Proc. $33^{\text {rd }}$ Conf. on Control. Fusion and Plasma Phys., Rome, 2006, Europhys. Conf. Abstr. 30I (2006) (P1.149).

[27] DOYLE, E.J., et al., Nucl. Fusion 47 (2007) S18.

[28] BEHN, R., et al., submitted to Plasma Phys. Control. Fusion (2014).

[29] GOHIL, P., et al., Nucl. Fusion 49 (2009) 115004.

[30] RYTER, F., and the H-Mode Threshold Database Group, Plasma Phys. Control. Fusion 44 (2002) A415.

[31] MCDONALD, D.C., et al., Plasma Phys. Control. Fusion 46 (2004) 519.

[32] FUNDAMENSKI, W., et al., Nucl. Fusion 52 (2012) 062003.

[33] MARTIN, Y., et al., Proc. $3^{\text {rd }}$ European Energy Conf., Budapest, 2013.

[34] WENNINGER, R.P., et al., Nucl. Fusion 53 (2013) 113004.

[35] NOWAK, S., et al., this conference, EX/P2-54.

[36] NOWAK, S., et al., AIP Conference Proceedings 1580 (2014) 502.

[37] NOWAK, S., et al., J. Phys.: Conf. Ser. 401 (2012) 012017.

[38] KAMLEITNER, J., et al., Proc. $40^{\text {th }}$ Conf. on Control. Fusion and Plasma Phys., Espoo, Finland, 2013, Europhys. Conf. Abstr. 37D (2013) (P5.127).

[39] COOPER, W.A., et al., Phys. Rev. Lett. 105 (2010) 035003.

[40] COOPER, W.A., et al., this conference, TH/P7-13.

[41] PORTE, L., et al., this conference, EX/P3-57.

[42] CODA, S., et al., Proc. $40^{\text {th }}$ Conf. on Control. Fusion and Plasma Phys., Espoo, Finland, 2013, Europhys. Conf. Abstr. 37D (2013) (P1.169).

[43] VUILlE, V., et al., Proc. $40^{\text {th }}$ Conf. on Control. Fusion and Plasma Phys., Espoo, Finland, 2013, Europhys. Conf. Abstr. 37D (2013) (P2.176).

[44] HENNEQUIN, P., et al., Nucl. Fusion 46 (2006) S771.

[45] CAMENEN, Y., et al., Nucl. Fusion 47 (2007) 510.

[46] PORTE, L., et al., Proc. $41^{\text {st }}$ Conf. on Control. Fusion and Plasma Phys., Berlin, 2014, Europhys. Conf. Abstr. 38F (2014) (P5.022).

[47] HUANG, Z., et al., Proc. $41^{\text {st }}$ Conf. on Control. Fusion and Plasma Phys., Berlin, 2014, Europhys. Conf. Abstr. 38F (2014) (P5.071).

[48] SAUTER, O., et al., Phys. Plasmas 21 (2014) 055906.

[49] DE MEIJERE, C.A., et al., Plasma Phys. Control. Fusion 56 (2014) 072001.

[50] WAHLBERG, C., et al., Plasma Phys. Control. Fusion 51 (2009) 085006.

[51] MARINI, C., et al., Proc. $41^{\text {st }}$ Conf. on Control. Fusion and Plasma Phys., Berlin, 2014, Europhys. Conf. Abstr. 38F (2014) (P5.023).

[52] STOLTZFUS-DUECK, T., et al., Phys. Plasmas 19 (2012) 055908.

[53] GOODMAN, T.P., et al, Phys. Rev. Lett. 106 (2011) 245002.

[54] MERLE, A., et al., this conference, EX/P3-55.

[55] KIRNEVA, N.A., et al., submitted to Plasma Phys. Control. Fusion (2014).

[56] KIRNEVA, N., et al., this conference, EX/P3-54.

[57] FEDERSPIEL, L.I., Ph.D. Thesis 6050, EPFL, Lausanne (2014).

[58] ARNOUX, G., et al., Nucl. Fusion 53 (2013) 073016.

[59] NESPOLI, F., et al., $21^{\text {st }}$ Int. PSI Conf., Ongaku-Do, Japan, submitted to J. Nucl. Mater. (2014).

[60] REIMERDES, H., et al., Plasma Phys. Control. Fusion 55 (2013) 124027.

[61] VIJVERS, W.A.J., et al., Nucl. Fusion 54 (2014) 023009.

[62] TAL, B., et al., Rev. Sci. Instrum. 84 (2013) 123508.

[63] LABIT, B., et al., Proc. $41^{\text {st }}$ Conf. on Control. Fusion and Plasma Phys., Berlin, 2014, Europhys. Conf. Abstr. 38F (2014) (P5.021).

[64] REIMERDES, H., et al., $21^{\text {st }}$ Int. PSI Conf., Ongaku-Do, Japan, submitted to J. Nucl. Mater. (2014).

[65] LUNT, T., et al., Plasma Phys. Control. Fusion 56 (2014) 035009.

[66] RYUTOV, D.D., et al., Contrib. Plasma Phys. 52 (2012) 538.

[67] CANAL, G.P., Ph.D. Thesis 6272, EPFL, Lausanne (2014).

[68] AMBROSINO, R., et al., submitted to Nucl. Fusion (2014).

[69] VIJVERS, W. et al., Proc. 55 ${ }^{\text {th }}$ DPP-APS Meeting, Denver, USA, Bull. Am. Phys. Soc. 58 (2013) PP8.00047. 


\section{Appendix: The TCV team}

R. Albanese ${ }^{\mathrm{a}}$, S.Alberti, R. Ambrosino ${ }^{\mathrm{a}}$, H. Anand, Y. Andrebe, M. Ariola ${ }^{\mathrm{a}}$, J.E. Barton ${ }^{\mathrm{b}}$, R.

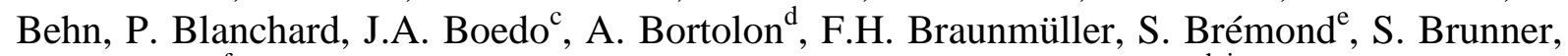
Y. Camenen ${ }^{\text {f }}$, G.P. Canal, S. Coda, W.A. Cooper, N. Cruz ${ }^{\mathrm{g}}$, M. de Baar ${ }^{\mathrm{h}, \mathrm{i}}$, J. Decker, C.A. de Meijere, B.P. Duval, A. Fasoli, L. Federspiel, F. Felici ${ }^{\text {h }}$, M. Fontana, I. Furno, T.P. Goodman, J.P. Graves, P. Hennequin ${ }^{j}$, J.-Ph. Hogge, G. Hommen ${ }^{\text {h,i }}$, Z. Huang, B. Joye, J. Kamleitner, A.N. Karpushov, D. Kim, N. Kirneva ${ }^{k, 1}$, A. Krämer-Flecken ${ }^{m}$, B. Labit, E. Lazzaron ${ }^{n}$, H.B. Le, L. Lefevre ${ }^{\mathrm{o}}$, F. Li, B. Lipschultz ${ }^{\mathrm{p}}$, J.B. Lister, X. Llobet, T. Lunt ${ }^{\mathrm{q}}$, E. Maljaars ${ }^{\mathrm{h}}$, C. Marini, Y. Martin, M. Mattei ${ }^{\mathrm{a}}$, A. Merle, P.A. Molina Cabrera, D. Moreau ${ }^{\mathrm{e}}$, J.-M. Moret, T. Morgan, N.A. Mustafin ${ }^{\mathrm{k}}$, F. Nespoli, R. Nouailletas ${ }^{\mathrm{e}}$, S. Nowak ${ }^{\mathrm{n}}$, Y. Peysson ${ }^{\mathrm{e}}$, A. Pironti ${ }^{\mathrm{a}}$, A. Pochelon, L. Porte, H. Reimerdes, O. Sauter, Ch. Schlatter, E. Schuster ${ }^{\mathrm{b}}$, M. Silva, J. Sinha, T. Stoltzfus-Dueck ${ }^{\mathrm{r}}$, B. Tál' , A.A. Teplukhina, D. Testa, C. Theiler, G. Tonetti, M.Q. Tran, C. Tsui $^{\mathrm{c}}$, L. Vermare ${ }^{\mathrm{j}}$, T. Vernay, W.A.J. Vijvers ${ }^{\mathrm{i}}$, N.M.T. Vu ${ }^{\mathrm{o}}$, V. Vuille, H. Weisen, R. Wenninger $^{\mathrm{q}}$, E. Witrant ${ }^{\mathrm{t}}$.

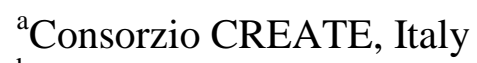

${ }^{\mathrm{b}}$ Lehigh University, US

${ }^{\mathrm{c} U C S D}, \mathrm{US}$

${ }^{\mathrm{d}} \mathrm{U}$. Tennessee, US

${ }^{\mathrm{e}} \mathrm{CEA}$, France

${ }^{\mathrm{f}} \mathrm{CNRS} / \mathrm{AMU}$, France

${ }^{\mathrm{g}}$ IST, Portugal

${ }^{\mathrm{h}}$ TUe, The Netherlands

i DIFFER, The Netherlands

${ }^{\mathrm{j}} \mathrm{LPP}$, France

${ }^{\mathrm{k}}$ RRC Kurchatov, Russian Federation

${ }^{1}$ NRNU MEPhI, Russian Federation

${ }^{\mathrm{m}}$ Forschungszentrum Jülich, Germany

${ }^{\mathrm{n}}$ IFP-CNR, Italy

${ }^{\circ}$ LCIS, Grenoble Institute of Technology, France

${ }^{\mathrm{p}}$ University of York, UK

${ }^{\mathrm{q}}$ IPP Garching, Germany

${ }^{\mathrm{r}}$ Princeton University, US

${ }^{\mathrm{s}}$ IPNP, Hungary

'Université de Grenoble, France 